\title{
UM ESTUDO CINÉTICO SOBRE A INFLUÊNCIA DE MEDIADORES FENÓLICOS NA DESCOLORAÇÃO DE DIFERENTES CORANTES POR SISTEMAS FENTON
}

\author{
Cássia Sidney Santana ${ }^{\mathrm{a}}$, Camila Cristina Vieira Velloso ${ }^{\mathrm{a}}$ e André Aguiar \\ aUniversidade Federal de São João del-Rei, Campus Alto Paraopeba, 36420-000 Ouro Branco - MG, Brasil \\ bUniversidade Federal de Itajubá, Instituto de Recursos Naturais, 37500-903 Itajubá - MG, Brasil
}

Recebido em 31/07/2018; aceito em 18/10/2018; publicado na web em 06/12/2018

\begin{abstract}
A KINETIC STUDY ABOUT THE INFLUENCE OF PHENOLIC MEDIATORS IN THE DECOLORIZATION OF DIFFERENT DYES BY FENTON SYSTEMS. The present work evaluated the influence of phenolic mediators (five dihydroxybenzenes and gallic acid) on the decolorization kinetics of phenol red, chromotrope $2 \mathrm{R}$, and methylene blue by Fenton systems. The reactions were performed with $\mathrm{H}_{2} \mathrm{O}_{2}$, iron salts as catalysts, and the absence or presence of phenolic mediators. Based on the decolorization data from previous study of our group, the $1^{\text {st }}$ and $2^{\text {nd }}$ order kinetic models were analyzed, as well as the alternative BMG model. It was found that the mediators accelerated the reactions, based in $1^{\text {st }}$ and $2^{\text {nd }}$ order kinetic models. Reactions containing $\mathrm{Fe}^{2+}$ fitted better to the BMG model, while reactions containing $\mathrm{Fe}^{3+}$ fitted better to the $2^{\text {nd }}$ order kinetic model, independent of mediators. Based on the three evaluated kinetic models, pro-oxidant properties were observed for the mediators in Fenton systems.
\end{abstract}

Keywords: Fenton reaction; hydroxyl radical; dihydroxybenzene; gallic acid; dye.

\section{INTRODUÇÃO}

Devido à limitação de técnicas convencionais para remoção ou degradação de poluentes orgânicos complexos como os corantes, presentes comumente em efluentes têxteis, têm-se buscado novas alternativas. Nos últimos anos têm-se destacado os processos oxidativos avançados (POAs), principalmente aqueles baseados na reação de Fenton para degradação de corantes ${ }^{1-3}$ A reação de Fenton consiste na degradação catalítica de peróxido de hidrogênio por íons ferrosos com a consequente formação in situ de radicais hidroxila $\left(\mathrm{HO}^{\bullet}\right.$; Eq. 1). Esses radicais possuem alto potencial padrão de redução $\left(\mathrm{E}^{\circ}=2,80 \mathrm{~V}\right)$, capazes de oxidar diferentes poluentes orgânicos a moléculas mais simples, inclusive convertê-los em $\mathrm{CO}_{2}, \mathrm{H}_{2} \mathrm{O}$ e íons inorgânicos. Essa reação é mais conhecida como reação de Fenton clássica. Os íons $\mathrm{Fe}^{3+}$ também podem ser utilizados em substituição ao $\mathrm{Fe}^{2+}$, pois os primeiros são mais abundantes, tendo-se a reação tipo Fenton (Eq. 2). Com base nas constantes cinéticas de ambas, a velocidade é menor na presença do $\mathrm{Fe}^{3+}$, além de gerar somente o radical hidroperoxila $\left(\mathrm{HO}_{2}{ }^{\bullet}\right)$, o qual possui potencial padrão de redução inferior ao $\mathrm{HO}^{\bullet} \cdot{ }^{4-6}$ Apesar de ser muito lenta, essa segunda reação é importante para reduzir $\mathrm{Fe}^{3+}$ a $\mathrm{Fe}^{2+}$ para reagir depois com $\mathrm{H}_{2} \mathrm{O}_{2}$ (Eq. 1). Na ausência de um substrato orgânico, o consumo de $\mathrm{HO}^{\bullet}$ envolve uma série de reações complexas concomitantemente (Eq. 3-6). ${ }^{6}$

$$
\begin{array}{ll}
\mathrm{Fe}^{2+}+\mathrm{H}_{2} \mathrm{O}_{2} \rightarrow \mathrm{Fe}^{3+}+\mathrm{HO}^{\bullet}+\mathrm{HO}^{-} & k=50-80 \mathrm{~mol}^{-1} 1 \mathrm{~s}^{-1} \\
\mathrm{Fe}^{3+}+\mathrm{H}_{2} \mathrm{O}_{2} \rightarrow \mathrm{Fe}^{2+}+\mathrm{HO}_{2}^{\bullet}+\mathrm{H}^{+} & k=0,002-0,01 \mathrm{~mol}^{-1} 1 \mathrm{~s}^{-1} \\
\mathrm{Fe}^{2+}+\mathrm{HO}^{\bullet} \rightarrow \mathrm{Fe}^{3+}+\mathrm{HO}^{-} & k=2,5-5 \times 10^{8} \mathrm{~mol}^{-1} 1 \mathrm{~s}^{-1} \\
\mathrm{HO}+\mathrm{HO}^{\bullet} \rightarrow \mathrm{H}_{2} \mathrm{O}_{2} & k=4-8 \times 10^{9} \mathrm{~mol}^{-1} 1 \mathrm{~s}^{-1} \\
\mathrm{H}_{2} \mathrm{O}_{2}+\mathrm{HO}^{\bullet} \rightarrow \mathrm{H}_{2} \mathrm{O}+\mathrm{HO}_{2} & k=1,7-4.5 \times 10^{7} \mathrm{~mol}^{-1} 1 \mathrm{~s}^{-1} \\
\mathrm{HO}^{\bullet}+\mathrm{HO}_{2} \rightarrow \mathrm{H}_{2} \mathrm{O}+\mathrm{O}_{2} & k=1,4 \times 10^{10} \mathrm{~mol}^{-1} 1 \mathrm{~s}^{-1}
\end{array}
$$

Uma forma de aumentar a geração de $\mathrm{HO} \bullet$ em sistemas Fenton e torná-la duradoura, independente do estado inicial de oxidação dos íons de ferro e da regeneração lenta de $\mathrm{Fe}^{2+}$ pela reação tipo Fenton, consiste na adição de mediadores fenólicos redutores. Diferentes

\footnotetext{
*e-mail: andrepiranga@yahoo.com.br; aguiar@unifei.edu.br
}

compostos que reduzem rápida e continuamente $\mathrm{Fe}^{3+}$ a $\mathrm{Fe}^{2+}$ têm sido estudados, sendo eles sintéticos ou de origem natural. ${ }^{4}$ Fenóis derivados de lignina como a vanilina, ${ }^{7,8}$ o metabólito fúngico ácido 3-hidroxiantranílico ${ }^{9}$ e o derivado de taninos ácido gálico têm sido avaliados como mediadores fenólicos naturais. ${ }^{10-12}$ Além deles, o aminoácido cisteína e o ácido ascórbico (não fenólicos), também têm proporcionado incrementos na degradação de corantes e outros poluentes orgânicos por sistemas Fenton. ${ }^{13,14}$ Por outro lado, compostos sintéticos como os di-hidroxibenzenos têm sido os mais testados, principalmente o catecol. ${ }^{15-17}$ Irradiação também pode promover a regeneração de íons $\mathrm{Fe}^{2+}$ para aumentar a produção de radicais $\mathrm{HO}^{\bullet}$. No entanto, o uso de reatores mais complexos, além do custo de energia elétrica adicional, limita a aplicação de sistemas foto-Fenton., ${ }^{5,6}$

Em estudos anteriores do nosso grupo têm sido observados incrementos na descoloração de corantes, pertencentes a diferentes classes, por sistemas Fenton devido à adição de diversos mediadores fenólicos redutores de $\mathrm{Fe}^{3+} \cdot{ }^{7-9,11,15}$ Entretanto, é preciso avaliar como esses compostos podem influenciar a velocidade de descoloração dos corantes baseado em diferentes modelos cinéticos. Alguns estudos têm-se limitado apenas ao modelo linear de $1^{\mathrm{a}}$ ordem para verificar o efeito pro-oxidante de mediadores. Percebe-se que esse modelo não é sempre o mais adequado, pois os valores de coeficientes de correlação que descrevem a cinética das reações $\left(\mathrm{r}^{2}\right)$ em alguns trabalhos são inferiores a 0,9 , o que pode comprometer a análise e a comparação de diferentes sistemas reacionais. ${ }^{13,14,18}$

Com base nessas considerações, o presente trabalho teve como objetivo realizar um estudo cinético baseado nos três modelos lineares mais estudados ( $1^{\mathrm{a}}$ ordem, $2^{\mathrm{a}}$ ordem e BMG) a partir de dados de um trabalho prévio do nosso grupo, ${ }^{15}$ envolvendo a descoloração de corantes de diferentes classes por sistemas Fenton homogêneos $\left(\mathrm{Fe}^{2+} / \mathrm{H}_{2} \mathrm{O}_{2}, \mathrm{Fe}^{3+} / \mathrm{H}_{2} \mathrm{O}_{2}\right)$. Os corantes avaliados foram o vermelho de fenol (trifenilmetilênico), cromotrope $2 \mathrm{R}$ (azo) e o azul de metileno (tiazina). Os experimentos de descoloração foram avaliados na ausência e na presença de cinco di-hidroxibenzenos: os ácidos 2,3-, 2,4-, 2,5-di-hidroxibenzóicos (DHBs) e 3,4-di-hidroxifenilacético (3,4-DHFA), além do catecol. O tri-hidroxibenzeno ácido gálico também foi avaliado como mediador. Pretende-se verificar nesse estudo o efeito pro-oxidante desses compostos fenólicos em sistemas Fenton. 


\section{PARTE EXPERIMENTAL}

Os reagentes utilizados nesse estudo foram de grau analítico e obtidos de diferentes fornecedores: cromotrope $2 \mathrm{R}$ e os ácidos 2,3-, 2,4-, 2,5-DHBs e 3,4-DHFA foram obtidos da Sigma-Aldrich (Milwaukee WI, Estados Unidos); catecol da Fluka Chemie (Buchs, Suiça); $\mathrm{Fe}\left(\mathrm{NO}_{3}\right)_{3}$, ácido gálico, vermelho de fenol e $\mathrm{H}_{2} \mathrm{O}_{2}(30 \%$ $\mathrm{m} / \mathrm{m}$ ) da Vetec Química (Rio de Janeiro, Brasil). Ácido sulfúrico e azul de metileno foram obtidos de outros fornecedores, mas também de grau analítico. As soluções foram preparadas com o uso de água deionizada e depois armazenadas adequadamente, com exceção das soluções de mediadores fenólicos que eram preparadas e usadas no mesmo dia, enquanto as soluções de sais de ferro eram preparadas imediatamente antes do uso, com o intuito de minimizar a alteração do estado de oxidação desse metal. As estruturas químicas dos cinco di-hidroxibenzenos e do ácido gálico, assim como as dos corantes, estão mostradas na Figura 1.

Para avaliar a descoloração dos corantes foram realizadas reações em cubetas de quartzo de $3 \mathrm{~mL}$ contendo $450 \mu \mathrm{mol} \mathrm{L}^{-1}$ de $\mathrm{H}_{2} \mathrm{O}_{2}$, $1 \mathrm{mmol} \mathrm{L}^{-1}$ de $\mathrm{H}_{2} \mathrm{SO}_{4}$ (para ajustar o $\mathrm{pH}$ na faixa ideal entre 2,5 e 3,0 para evitar precipitação de ferro - medido com o auxílio de um pHmetro), $30 \mu \mathrm{mol} \mathrm{L}-1$ de corante e $30 \mu \mathrm{mol} \mathrm{L}{ }^{-1}$ de $\mathrm{Fe}\left(\mathrm{NO}_{3}\right)_{3}$ ou $\mathrm{FeSO}_{4}$ para iniciar os tratamentos baseados nas reações tipo Fenton ou Fenton clássica, respectivamente. Quando realizados os experimentos na presença de mediadores fenólicos nas reações tipo Fenton, soluções contendo os mesmos foram adicionadas ao meio reacional para uma concentração final de $10 \mu \mathrm{mol} \mathrm{L}{ }^{-1}$. Nenhum dos reagentes foi adicionado ao longo do tempo reacional. $\mathrm{O}$ volume final para cada reação correspondeu a $2 \mathrm{~mL}$. Leituras de absorbância foram realizadas no comprimento de onda máximo característico de cada corante $(435$, 513 e $665 \mathrm{~nm}$, para o vermelho de fenol, cromotrope 2R e azul de metileno, respectivamente) por meio de um espectrofotômetro Libra S50 (Biochrom). A descoloração dos corantes foi monitorada após 5, 10, 20, 40 e $60 \mathrm{~min}$. Preparou-se o branco isento de corantes e sais de ferro. Os ensaios foram realizados em duplicata, ao abrigo da luz e à temperatura ambiente. Mais detalhes das condições experimentais estão reportados em Barreto et al. ${ }^{15}$ As porcentagens de descoloração dos corantes foram determinadas conforme Equação 7:

$$
\% \text { Descoloração }=\left(1-\mathrm{C}_{\mathrm{t}} / \mathrm{C}_{0}\right) \times 100 \%
$$

sendo $\mathrm{C}_{0}$ e $\mathrm{C}_{\mathrm{t}}$ as concentrações inicial e no tempo t do corante, respectivamente. As concentrações residuais dos corantes foram determinadas com o auxílio de curvas de calibração preparadas na presença de $1 \mathrm{mmol} \mathrm{L}^{-1}$ de $\mathrm{H}_{2} \mathrm{SO}_{4}$, para evitar alterações no comprimento de onda máximo de cada corante.

Com o intuito de se avaliar a influência dos mediadores fenólicos nas reações de descoloração, realizou-se um estudo cinético a partir dos modelos de $1^{\text {a }}$ e $2^{\mathrm{a}}$ ordens conforme Equações 8 e 9 , respectivamente, ${ }^{19}$ além do modelo desenvolvido por Behnajady, Modirshahla e Ghanbary - modelo BMG ${ }^{20}$ conforme Equação 10 :

$$
\begin{gathered}
\ln \left(\mathrm{C}_{0} / \mathrm{C}_{\mathrm{t}}\right)=k_{1} \cdot \mathrm{t} \\
1 / \mathrm{C}_{\mathrm{t}}=1 / \mathrm{C}_{0}+k_{2} \cdot \mathrm{t} \\
\mathrm{t} /\left(1-\mathrm{C}_{\mathrm{l}} / \mathrm{C}_{0}\right)=m+b . \mathrm{t}
\end{gathered}
$$

sendo $k_{1}$ e $k_{2}$ as constantes cinéticas obtidas para os modelos de $1^{\text {a }}$ e $2^{a}$ ordens. Os valores de tempo de meia vida $\left(t_{1 / 2}\right)$ também foram calculados para os dois modelos. A partir das duas constantes características do modelo BMG, $m$ e $b$, pôde-se calcular seus inversos para estimar a velocidade inicial $(1 / m)$ e a capacidade oxidativa $(1 / b)$ das reações, respectivamente. ${ }^{20}$ As unidades de todas as constantes estão descritas nas Tabelas 1 e 2, exceto $b$ que é adimensional.

\section{RESULTADOS E DISCUSSÃO}

Curvas contendo alguns dos dados de descoloração são mostradas nas Figuras 2 e 3 para os três diferentes modelos cinéticos testados. Baseado no modelo de $1^{\mathrm{a}}$ ordem, a Figura 2 a mostra os dados de descoloração do vermelho de fenol por reação tipo Fenton na ausência<smiles>O=C(O)c1cccc(O)c1O</smiles>

Ácido 2,3-DHB<smiles>Oc1ccccc1O</smiles>

Catecol<smiles>O=C(O)c1cc(O)ccc1O</smiles><smiles>O=C(O)Cc1ccc(O)c(O)c1</smiles>

Ácido 3,4-DHFA
Ácido 2,5-DHB<smiles>O=C(O)c1ccc(O)cc1O</smiles>

Ácido 2,4-DHB<smiles>C1CCC1</smiles><smiles>O=C(O)c1cc(O)c(O)c(O)c1</smiles>

Ácido gálico

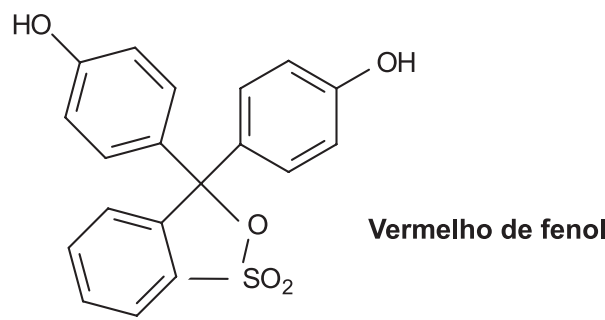<smiles>O=S(=O)(O[Na])c1cc(O)c2c(O)c(N=Nc3ccccc3)c(S(=O)(=O)O[Na])cc2c1</smiles>

Cromotrope 2R<smiles>CN(C)c1ccc2nc3ccc(N(C)C)cc3[s+]c2c1</smiles>

Azul de metileno

Figura 1. Estrutura química dos mediadores fenólicos e dos três corantes avaliados no presente estudo 
e na presença de três dos mediadores fenólicos avaliados. A presença desses mediadores aumentou a inclinação das curvas, indicando aumento nos valores de $k_{1}$ e consequentemente na velocidade de descoloração. O ácido 2,5-DHB foi o mais efetivo em aumentar a inclinação, seguido pelo ácido 3,4-DHFA, enquanto o catecol foi o menos efetivo entre os três mediadores. Da mesma forma, a Figura $2 b$ apresenta as curvas de descoloração do mesmo corante por $\mathrm{Fe}^{3+} / \mathrm{H}_{2} \mathrm{O}_{2}$ com base no modelo de $2^{\mathrm{a}}$ ordem, para as reações na ausência e na presença de três mediadores. Nesse caso, também são observados aumentos na inclinação das curvas, ou seja, aumentos de $k_{2}$, com destaque para os ácidos 2,3-e 2,5-DHBs, sendo que ambos apresentaram comportamentos bastante similares. $\mathrm{O}$ ácido gálico promoveu uma menor inclinação em comparação aos dois isômeros mencionados.

Os valores de $k, \mathrm{r}^{2} \mathrm{e} \mathrm{t}_{1 / 2}$ obtidos a partir dos modelos de $1^{\mathrm{a}} \mathrm{e}$ $2^{\mathrm{a}}$ ordens estão mostrados na Tabela 1 para os três corantes avaliados. Os valores médios de $\mathrm{r}^{2}$ foram calculados, pois eles podem indicar qual é o modelo cinético mais apropriado para um determinado conjunto de reações. ${ }^{21,22}$

Para o corante vermelho de fenol, a reação $\mathrm{Fe}^{3+} / \mathrm{H}_{2} \mathrm{O}_{2}$ foi descrita pelos modelos de $1^{\mathrm{a}}$ e $2^{\mathrm{a}}$ ordens, enquanto o oposto foi observado para $\mathrm{Fe}^{2+} / \mathrm{H}_{2} \mathrm{O}_{2}$ (valores de $\mathrm{r}^{2}$ inferiores a 0,54). As reações $\mathrm{Fe}^{3+} / \mathrm{H}_{2} \mathrm{O}_{2} /$ mediadores fenólicos também foram bem descritas pelo modelo de $1^{\mathrm{a}}$ ordem, mas melhor descritas pelo de $2^{\mathrm{a}}$ ordem baseado nos valores médios de $\mathrm{r}^{2}$. Os mediadores aceleraram a descoloração desse corante com base nos aumentos de $k_{1} e k_{2}$. Como não houve descoloração do vermelho de fenol por $\mathrm{Fe}^{3+} / \mathrm{H}_{2} \mathrm{O}_{2}$ na presença do ácido 2,4-DHB, não foi possível fazer um estudo cinético dessa reação. Esse fenol é o único que não possui propriedades redutoras dentre os seis estudados. Seus isômeros com propriedades redutoras, os ácidos 2,3- e 2,5-DHBs, foram os mais efetivos com base nos maiores valores de $k_{1}, k_{2}$ e nas porcentagens de descoloração após $60 \mathrm{~min}$. Para os valores de $\mathrm{t}_{1 / 2}$, verificou-se redução de 22 vezes nesse parâmetro para $\mathrm{Fe}^{2+} / \mathrm{H}_{2} \mathrm{O}_{2}$ em relação à reação $\mathrm{Fe}^{3+} / \mathrm{H}_{2} \mathrm{O}_{2}$. Esses resultados são esperados, pois a reação de Fenton gera diretamente radicais $\mathrm{HO}^{\bullet}$ e em maior quantidade em comparação à reação tipo Fenton, a qual depende da redução lenta de $\mathrm{Fe}^{3+}$ por $\mathrm{H}_{2} \mathrm{O}_{2} \cdot{ }^{4,5} \mathrm{~A}$ utilização dos mediadores, exceto o ácido 2,4-DHB, reduziu cerca de $90 \%$ no $\mathrm{t}_{1 / 2}$ ao comparar com a reação na ausência deles $\left(\mathrm{Fe}^{3+} / \mathrm{H}_{2} \mathrm{O}_{2}\right)$. Importante mencionar também que as reações $\mathrm{Fe}^{3+} / \mathrm{H}_{2} \mathrm{O}_{2}$ na presença dos ácidos 2,3- e 2,5-DHBs podem ser bem mais efetivas que $\mathrm{Fe}^{2+} / \mathrm{H}_{2} \mathrm{O}_{2}$ para descolorir o vermelho de fenol, de acordo com os parâmetros cinéticos calculados.

Considerando os dados de descoloração do corante cromotrope $2 \mathrm{R}$, os modelos de $1^{\mathrm{a}}$ e $2^{\mathrm{a}}$ ordens apresentaram valores de $\mathrm{r}^{2}$ inferiores



a 0,9 para $\mathrm{Fe}^{3+} / \mathrm{H}_{2} \mathrm{O}_{2}$, enquanto o de $2^{\text {a }}$ ordem foi adequado para $\mathrm{Fe}^{2+} /$ $\mathrm{H}_{2} \mathrm{O}_{2}$. Os valores de $k_{1}$ e $k_{2}$ para $\mathrm{Fe}^{3+} / \mathrm{H}_{2} \mathrm{O}_{2} /$ mediadores fenólicos foram superiores, inclusive ao comparar com a reação de Fenton clássica, e promoveram maior descoloração do corante. O ácido 2,5-DHB também foi o mais efetivo para descolorir esse corante, pois na presença dele foram observados maiores valores de $k$. Menores valores de $\mathrm{t}_{1 / 2}$ foram observados para $\mathrm{Fe}^{2+} / \mathrm{H}_{2} \mathrm{O}_{2}$ em relação ao $\mathrm{Fe}^{3+} / \mathrm{H}_{2} \mathrm{O}_{2}$ também para esse corante. Na presença de mediadores o $t_{1 / 2}$ foi aproximadamente 9 vezes menor para o modelo de $1^{\text {a }}$ ordem e 43 vezes menor para o modelo de $2^{\mathrm{a}}$ ordem em relação à reação sem mediadores. Ao comparar com a reação de Fenton clássica, as reações $\mathrm{Fe}^{3+} / \mathrm{H}_{2} \mathrm{O}_{2}$ /mediadores fenólicos também resultaram consideravelmente em menores $\mathrm{t}_{1 / 2}$, baseando-se nesses dois modelos cinéticos. A reação com o ácido 2,4-DHB permitiu aumentar os valores de $k_{1}$ e $k_{2}$, assim como diminuir o $t_{1 / 2}$ somente para o corante cromotrope $2 \mathrm{R}$. Por apresentar seus grupos hidroxila meta orientados (um grupo $\mathrm{OH}$ em relação ao outro no anel aromático), esse composto não reduz $\mathrm{Fe}^{3+}$. Contrariamente, os outros mediadores possuem propriedades redutoras, pois seus grupos hidroxila estão orientados nas posições orto ou para, podendo reduzir $\mathrm{Fe}^{3+}$ e serem oxidados em suas respectivas quinonas. ${ }^{23}$ Todavia, o ácido 2,4-DHB pode ser hidroxilado por radicais $\mathrm{HO}^{\bullet}$ durante os ensaios, sendo convertido em compostos para- ou orto-di-hidroxiaromáticos com propriedades redutoras. ${ }^{11,15} \mathrm{Em}$ um estudo anterior, Chen et al. observaram que o ácido salicílico (2-hidroxibenzóico) apresentou propriedades pro-oxidantes ao ser hidroxilado durante a descoloração do corante verde de malaquita por $\mathrm{Fe}^{3+} / \mathrm{H}_{2} \mathrm{O}_{2} .{ }^{24}$

Para o azul de metileno, a reação $\mathrm{Fe}^{2+} / \mathrm{H}_{2} \mathrm{O}_{2}$ se adequou muito bem ao modelo de $1^{\text {a }}$ ordem, corroborando a literatura. ${ }^{25-27}$ Os sistemas reacionais $\mathrm{Fe}^{3+} / \mathrm{H}_{2} \mathrm{O}_{2} /$ mediadores fenólicos redutores foram melhor descritos pelo modelo de $2^{\mathrm{a}}$ ordem, enquanto o valor médio de $\mathrm{r}^{2}$ para $1^{\text {a }}$ ordem foi inferior a 0,9 . Os valores de $k_{1}$ e $k_{2}$ tiveram incrementos na presença dos mediadores. O modelo de $2^{\mathrm{a}}$ ordem não foi apropriado para as reações $\mathrm{Fe}^{3+} / \mathrm{H}_{2} \mathrm{O}_{2}$ e Fe ${ }^{3+} / \mathrm{H}_{2} \mathrm{O}_{2} /$ ácido 2,4-DHB $\left(\mathrm{r}^{2}\right.$ inferior a $0,8)$. Nas reações contendo os mediadores redutores, para ambos os modelos, os tempos de meia vida apresentaram valores próximos ao da reação $\mathrm{Fe}^{2+} / \mathrm{H}_{2} \mathrm{O}_{2}$. Os menores valores de $\mathrm{t}_{1 / 2}$ foram observados para as reações contendo os ácidos 2,3- e 2,5-DHBs. A presença do ácido 2,4-DHB praticamente não afetou de forma significativa os valores de $k_{l}, k_{2} \mathrm{e} \mathrm{t}_{1 / 2}$. Entretanto, a descoloração desse corante por todas as reações atingiu valores superiores a $90 \%$, inclusive na presença do ácido 2,4-DHB. Esses aspectos podem ser devido à alta suscetibilidade desse corante ao ataque por radicais $\mathrm{HO}^{\bullet},{ }^{7}$ dificultando a diferenciação das reações em função da porcentagem de descoloração após 60 min.

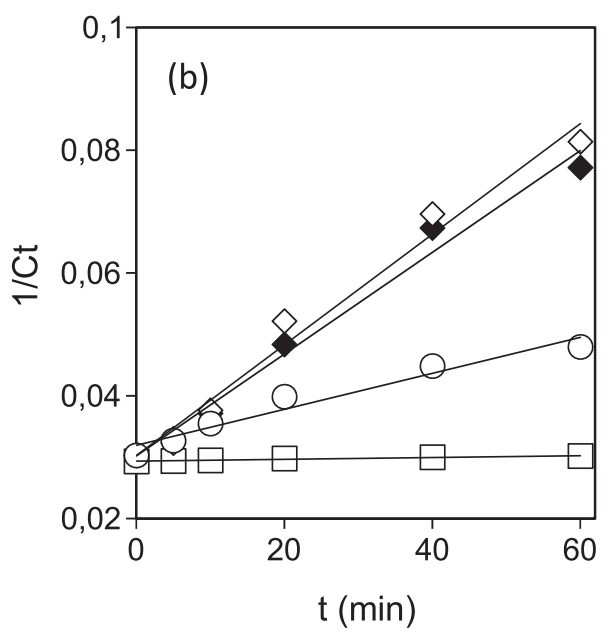

Figura 2. Curvas obtidas para a descoloração do corante vermelho de fenol a partir do modelo de $1^{a}$ ordem (a) por: $\mathrm{Fe}^{3+} / \mathrm{H}_{2} \mathrm{O}_{2}(\square), \mathrm{Fe}^{3+} / \mathrm{H}_{2} \mathrm{O}_{2} /$ catecol $(\bullet)$,

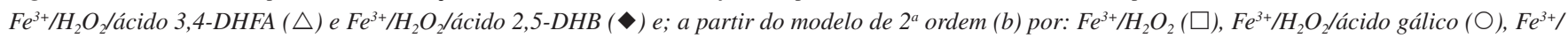

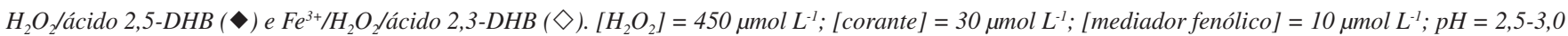


Tabela 1. Diferentes sistemas reacionais avaliados e as respectivas porcentagens de descoloração de cada corante após 60 min, ${ }^{15}$ constantes cinéticas dos modelos de $1^{\mathrm{a}}\left(k_{1}\right)$ e $2^{\mathrm{a}}$ ordens $\left(k_{2}\right)$, tempo de meia vida $\left(\mathrm{t}_{1 / 2}\right)$ e os coeficientes de correlação $\left(\mathrm{r}^{2}\right)$ obtidos após os ajustes dos dados

\begin{tabular}{|c|c|c|c|c|c|c|c|}
\hline Sistema reacional & \% de descoloração & $\begin{array}{c}k_{1} \\
\left(\min ^{-1}\right)\end{array}$ & $\begin{array}{c}\mathrm{t}_{1 / 2} \\
(\mathrm{~min})\end{array}$ & $\begin{array}{c}\mathrm{r}^{2} \\
\left(1^{\mathrm{a}} \text { ordem }\right)\end{array}$ & $\begin{array}{c}k_{2} \\
\left(\mu \mathrm{mol}^{-1} \mathrm{~L} \mathrm{~min}^{-1}\right)\end{array}$ & $\mathrm{t}_{1 / 2}(\min )$ & $\begin{array}{c}r^{2} \\
\left(2^{a} \text { ordem }\right)\end{array}$ \\
\hline \multicolumn{8}{|l|}{ Vermelho de Fenol } \\
\hline $\mathrm{Fe}^{2+} / \mathrm{H}_{2} \mathrm{O}_{2}$ & $41,1 \pm 0,1$ & 0,0110 & 63,0134 & 0,5326 & 0,0002 & 147,9018 & 0,4066 \\
\hline \multicolumn{8}{|l|}{$\mathrm{Fe}^{3+} / \mathrm{H}_{2} \mathrm{O}_{2}$} \\
\hline Sem fenóis & $2,9 \pm 0,1$ & 0,0005 & 1386,2944 & 0,9467 & 0,00001 & 2930,2420 & 0,9575 \\
\hline Ácido 2,3-DHB & $62,8 \pm 1,2$ & 0,0185 & 37,4674 & 0,9354 & 0,0009 & 33,6654 & 0,9806 \\
\hline Ácido 2,4-DHB & $0,0 \pm 0,0$ & - & - & - & - & - & - \\
\hline Ácido 2,5-DHB & $60,7 \pm 0,2$ & 0,0174 & 39,8360 & 0,9498 & 0,0008 & 37,8736 & 0,9842 \\
\hline Ácido 3,4-DHFA & $35,6 \pm 1,8$ & 0,0072 & 96,2704 & 0,9966 & 0,0003 & 101,1928 & 0,9909 \\
\hline Ácido Gálico & $36,8 \pm 0,4$ & 0,0089 & 77,8817 & 0,8657 & 0,0003 & 100,9962 & 0,9516 \\
\hline Catecol & $17,1 \pm 0,6$ & 0,0031 & 223,5959 & 0,9984 & 0,0001 & 308,3817 & 0,9988 \\
\hline Média para as reações com fenóis & & & & 0,9492 & & & 0,9812 \\
\hline \multicolumn{8}{|l|}{ Cromotrope $2 \mathrm{R}$} \\
\hline $\mathrm{Fe}^{2+} / \mathrm{H}_{2} \mathrm{O}_{2}$ & $72,6 \pm 0,6$ & 0,0263 & 26,3554 & 0,3570 & 0,0014 & 25,7431 & 0,9180 \\
\hline \multicolumn{8}{|l|}{$\mathrm{Fe}^{3+} / \mathrm{H}_{2} \mathrm{O}_{2}$} \\
\hline Sem fenóis & $27,0 \pm 0,1$ & 0,0056 & 123,7763 & 0,8156 & 0,0002 & 170,0016 & 0,8850 \\
\hline Ácido 2,3-DHB & $94,5 \pm 0,0$ & 0,0563 & 12,3117 & 0,8065 & 0,0098 & 3,4694 & 0,9952 \\
\hline Ácido 2,4-DHB & $92,6 \pm 0,1$ & 0,0448 & 15,4720 & 0,9929 & 0,0070 & 4,8572 & 0,9455 \\
\hline Ácido 2,5-DHB & $95,1 \pm 0,1$ & 0,0582 & 11,9097 & 0,8006 & 0,0109 & 3,1193 & 0,9926 \\
\hline Ácido 3,4-DHFA & $93,5 \pm 0,2$ & 0,0507 & 13,6715 & 0,9300 & 0,0082 & 4,1464 & 0,9819 \\
\hline Ácido Gálico & $94,2 \pm 0,3$ & 0,0535 & 12,9560 & 0,8813 & 0,0092 & 3,6957 & 0,9809 \\
\hline Catecol & $94,7 \pm 0,1$ & 0,0544 & 12,7417 & 0,9153 & 0,0100 & 3,4000 & 0,9753 \\
\hline Média para as reações com fenóis & & & & 0,8878 & & & 0,9786 \\
\hline \multicolumn{8}{|l|}{ Azul de Metileno } \\
\hline $\mathrm{Fe}^{2+} / \mathrm{H}_{2} \mathrm{O}_{2}$ & $96,4 \pm 0,3$ & 0,0578 & 11,9922 & 0,9884 & 0,0166 & 2,2890 & 0,9167 \\
\hline \multicolumn{8}{|l|}{$\mathrm{Fe}^{3+} / \mathrm{H}_{2} \mathrm{O}_{2}$} \\
\hline Sem fenóis & $93,1 \pm 0,4$ & 0,0359 & 19,3077 & 0,8490 & 0,0068 & 5,0145 & 0,7378 \\
\hline Ácido 2,3-DHB & $97,2 \pm 0,4$ & 0,0705 & 9,8319 & 0,9000 & 0,0246 & 1,4654 & 0,9331 \\
\hline Ácido 2,4-DHB & $92,5 \pm 2,0$ & 0,0347 & 19,9754 & 0,8446 & 0,0065 & 5,4776 & 0,7452 \\
\hline Ácido 2,5-DHB & $97,4 \pm 0,1$ & 0,0750 & 9,2420 & 0,7294 & 0,0240 & 1,4630 & 0,9643 \\
\hline Ácido 3,4-DHFA & $96,6 \pm 0,1$ & 0,0664 & 10,4390 & 0,8812 & 0,0174 & 1,9298 & 0,9735 \\
\hline Ácido Gálico & $95,9 \pm 0,1$ & 0,0610 & 11,3631 & 0,9335 & 0,0140 & 2,3142 & 0,9709 \\
\hline Catecol & $96,6 \pm 0,0$ & 0,0604 & 11,4759 & 0,9717 & 0,0175 & 2,0065 & 0,9477 \\
\hline Média para as reações com fenóis & & & & 0,8767 & & & 0,9225 \\
\hline
\end{tabular}

Porém, com base nesses dois modelos cinéticos, foi possível verificar de forma mais clara o efeito pro-oxidante de compostos redutores de $\mathrm{Fe}^{3+}$ na descoloração do azul de metileno.

Quanto ao terceiro modelo avaliado no presente trabalho, BMG, sua sigla foi criada em função das iniciais dos três autores que o utilizaram pela primeira vez em reações para descolorir um corante por reação de Fenton. ${ }^{20} \mathrm{~A}$ tangente da curva de decréscimo em um determinado tempo para o modelo BMG pode ser obtida ao derivar a Equação 10, resultando na Equação 11:

$$
\left(\mathrm{dC}_{\mathrm{t}} / \mathrm{C}_{0}\right) / \mathrm{dt}=-m /(m+b \cdot \mathrm{t})^{2}
$$

Dessa forma, quanto maior for $1 / m$ (na condição em que $t$ se aproxima de 0 ), mais rápida será a razão de decréscimo do corante.
Sendo assim, 1/m indica a velocidade inicial de descoloração do corante. Quando o tempo for alto e tender ao infinito, o inverso de $b(1 / b)$ indica a fração máxima teórica de remoção, a qual equivale à capacidade máxima de oxidação do sistema ao final da reação.

A Figura 3 mostra as curvas obtidas a partir do modelo BMG para a descoloração do cromotrope $2 \mathrm{R}$ pelos sistemas $\mathrm{Fe}^{2+} / \mathrm{H}_{2} \mathrm{O}_{2}$, $\mathrm{Fe}^{3+} / \mathrm{H}_{2} \mathrm{O}_{2}$ e $\mathrm{Fe}^{3+} / \mathrm{H}_{2} \mathrm{O}_{2}$ /ácido 2,5-DHB. A última reação apresentou maior capacidade oxidativa devido a menor inclinação da curva, demonstrando o efeito pro-oxidante do ácido 2,5-DHB. Também é possível observar uma menor inclinação da curva referente à reação $\mathrm{Fe}^{2+} / \mathrm{H}_{2} \mathrm{O}_{2}$ em relação à $\mathrm{Fe}^{3+} / \mathrm{H}_{2} \mathrm{O}_{2}$, pois a primeira gera radicais $\mathrm{HO}^{\bullet}$ diretamente. Os dados extraídos desse modelo, assim como os coeficientes de correlação das curvas que descrevem a cinética de cada reação estão mostrados na Tabela 2 . 


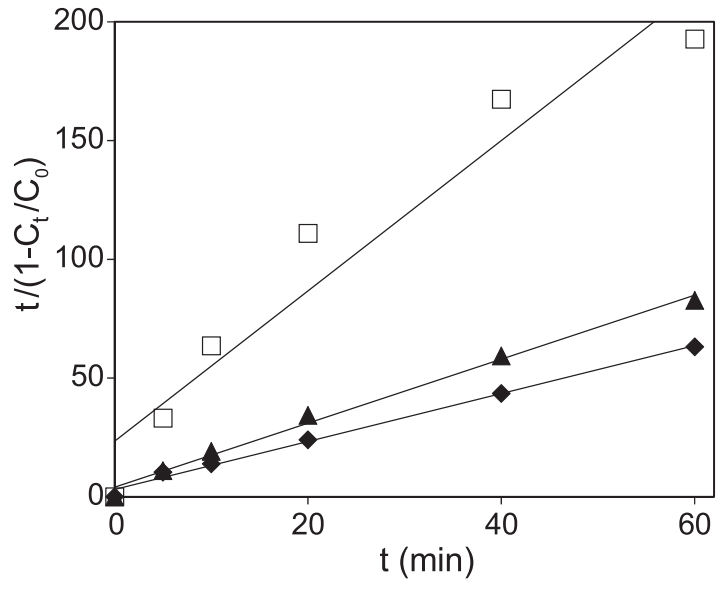

Figura 3. Curvas obtidas para o modelo $B M G$ a partir de dados de descoloração do corante cromotrope $2 \mathrm{R}$ por: $\mathrm{Fe}^{3+} / \mathrm{H}_{2} \mathrm{O}_{2} / a ́ c i d o ~ 2,5-\mathrm{DHB}(\bullet), \mathrm{Fe}^{2+} /$ $\mathrm{H}_{2} \mathrm{O}_{2}(\mathbf{\Delta}) e \mathrm{Fe}^{3+} / \mathrm{H}_{2} \mathrm{O}_{2}(\square)$
Valores de $\mathrm{r}^{2}$ acima de 0,99 revelam que o modelo BMG representa bem os dados experimentais para os três corantes descoloridos por $\mathrm{Fe}^{2+} / \mathrm{H}_{2} \mathrm{O}_{2}$. Anteriormente, a descoloração de diferentes corantes do tipo azo por $\mathrm{Fe}^{2+} / \mathrm{H}_{2} \mathrm{O}_{2}$ também se adequou bem a esse modelo, ${ }^{20,22,28}$ além de corantes de outras classes como a rodamina $\mathrm{B}$ e o próprio azul de metileno. ${ }^{3}$

Para a descoloração do corante vermelho de fenol na presença dos sistemas reacionais com mediadores, somente a reação com o ácido gálico foi bem descrita pelo modelo BMG, sendo esse mais apropriado que os outros modelos convencionais para essa reação. Ao comparar as reações $\mathrm{Fe}^{2+} / \mathrm{H}_{2} \mathrm{O}_{2}$ e Fe ${ }^{3+} / \mathrm{H}_{2} \mathrm{O}_{2}$ /ácido gálico a segunda apresentou maior capacidade oxidativa, mas menor velocidade inicial. Valores negativos de $b$ foram observados para duas das reações com mediadores, indicando a falta de ajuste desse modelo.

Para $\mathrm{Fe}^{3+} / \mathrm{H}_{2} \mathrm{O}_{2}$, apenas a descoloração do cromotrope $2 \mathrm{R}$ foi descrita por esse modelo cinético $\left(\mathrm{r}^{2}\right.$ maior que 0,9$)$. Os inversos de $m$ e $b$ aumentaram na presença dos mediadores, ou seja, eles aumentaram a velocidade inicial de reação, assim como a capacidade

Tabela 2. Parâmetros cinéticos obtidos a partir do modelo BMG $(m, b)$ para os diferentes sistemas reacionais avaliados, o inverso dos mesmos e os respectivos coeficientes de correlação $\left(\mathrm{r}^{2}\right)$

\begin{tabular}{|c|c|c|c|c|c|}
\hline Sistema reacional & $m(\min )$ & $1 / m\left(\min ^{-1}\right)$ & $b$ & $1 / b$ & $r^{2}$ \\
\hline \multicolumn{6}{|l|}{ Vermelho de Fenol } \\
\hline $\mathrm{Fe}^{2+} / \mathrm{H}_{2} \mathrm{O}_{2}$ & 3,1958 & 0,3129 & 2,3979 & 0,4170 & 0,9996 \\
\hline \multicolumn{6}{|l|}{$\mathrm{Fe}^{3+} / \mathrm{H}_{2} \mathrm{O}_{2}$} \\
\hline Sem fenóis & 1556,6753 & 0,0006 & 6,2946 & 0,1589 & 0,2403 \\
\hline Ácido 2,3-DHB & 55,5896 & 0,0180 & 0,5142 & 1,9448 & 0,3376 \\
\hline Ácido 2,4-DHB & - & - & - & - & - \\
\hline Ácido 2,5-DHB & 48,3172 & 0,0207 & 0,7409 & 1,3497 & 0,8171 \\
\hline Ácido 3,4-DHFA & 177,3000 & 0,0056 & $-0,2423$ & $-4,1271$ & 0,2444 \\
\hline Ácido Gálico & 53,0663 & 0,0188 & 1,7885 & 0,5591 & 0,9870 \\
\hline Catecol & 409,2443 & 0,0024 & $-1,3862$ & $-0,7214$ & 0,3777 \\
\hline Média para as reações com fenóis & & & & & 0,5528 \\
\hline \multicolumn{6}{|l|}{ Cromotrope $2 \mathrm{R}$} \\
\hline $\mathrm{Fe}^{2+} / \mathrm{H}_{2} \mathrm{O}_{2}$ & 6,3220 & 0,1582 & 1,2947 & 0,7724 & 0,9969 \\
\hline \multicolumn{6}{|l|}{$\mathrm{Fe}^{3+} / \mathrm{H}_{2} \mathrm{O}_{2}$} \\
\hline Sem fenóis & 36,3233 & 0,0275 & 2,8603 & 0,3496 & 0,9383 \\
\hline Ácido 2,3-DHB & 5,3152 & 0,1881 & 0,9648 & 1,0365 & 0,9992 \\
\hline Ácido 2,4-DHB & 23,4309 & 0,0427 & 0,6512 & 1,5356 & 0,9520 \\
\hline Ácido 2,5-DHB & 4,8052 & 0,2081 & 0,9692 & 1,0318 & 0,9995 \\
\hline Ácido 3,4-DHFA & 9,1497 & 0,1093 & 0,9082 & 1,1011 & 0,9990 \\
\hline Ácido Gálico & 6,5674 & 0,1523 & 0,9501 & 1,0525 & 0,9998 \\
\hline Catecol & 7,7544 & 0,1290 & 0,9205 & 1,0864 & 0,9989 \\
\hline Média para as reações com fenóis & & & & & 0,9914 \\
\hline \multicolumn{6}{|l|}{ Azul de Metileno } \\
\hline $\mathrm{Fe}^{2+} / \mathrm{H}_{2} \mathrm{O}_{2}$ & 11,3642 & 0,0880 & 0,8414 & 1,1885 & 0,9945 \\
\hline \multicolumn{6}{|l|}{$\mathrm{Fe}^{3+} / \mathrm{H}_{2} \mathrm{O}_{2}$} \\
\hline Sem fenóis & 187,3135 & 0,0053 & $-2,3736$ & $-0,4213$ & 0,8651 \\
\hline Ácido 2,3-DHB & 19,9081 & 0,0502 & 0,6122 & 1,6335 & 0,6966 \\
\hline Ácido 2,4-DHB & 160,0154 & 0,0062 & $-1,5651$ & $-0,6389$ & 0,0938 \\
\hline Ácido 2,5-DHB & 3,9325 & 0,2543 & 0,9480 & 1,0549 & 0,9963 \\
\hline Ácido 3,4-DHFA & 7,7010 & 0,1299 & 0,8831 & 1,1324 & 0,9907 \\
\hline Ácido Gálico & 14,2793 & 0,0700 & 0,7573 & 1,3205 & 0,9486 \\
\hline Catecol & 23,7353 & 0,0421 & 0,5685 & 1,7590 & 0,8580 \\
\hline Média para as reações com fenóis & & & & & 0,8980 \\
\hline
\end{tabular}


oxidativa, respectivamente. Ao comparar com Fe ${ }^{2+} / \mathrm{H}_{2} \mathrm{O}_{2}$, os sistemas reacionais $\mathrm{Fe}^{3+} / \mathrm{H}_{2} \mathrm{O}_{2} /$ mediadores ainda apresentaram maior capacidade oxidativa, enquanto a velocidade inicial foi maior apenas para as reações na presença dos ácidos 2,3- ou 2,5-DHBs. Apesar de a reação com o ácido 2,4-DHB apresentar uma baixa velocidade inicial, foi observada uma alta capacidade oxidativa para a mesma para descolorir o cromotrope $2 \mathrm{R}$.

Para o azul de metileno, o modelo BMG foi o mais adequado para $\mathrm{Fe}^{2+} / \mathrm{H}_{2} \mathrm{O}_{2}$. Para as reações que descoloriram menos o corante, $\mathrm{Fe}^{3+} / \mathrm{H}_{2} \mathrm{O}_{2}$ e $\mathrm{Fe}^{3+} / \mathrm{H}_{2} \mathrm{O}_{2}$ /ácido 2,4-DHB, foram observados valores negativos de $b$, indicando a falta de ajuste desse modelo. Ao comparar os quatro sistemas reacionais com $\mathrm{r}^{2}$ maior que 0,94 para o modelo BMG, a velocidade inicial de descoloração foi maior para aqueles contendo mediadores. No entanto, ao contrário dos ácidos 2,5-DHB e 3,4-DHFA, a reação contendo o ácido gálico apresentou menor velocidade inicial ao comparar com a reação de Fenton clássica. O ácido gálico foi o único dos mediadores em que todas as reações na sua presença se adequaram bem ao modelo BMG, inclusive para a descoloração do alaranjado de metila em um estudo previamente publicado. ${ }^{22}$

O azul de metileno é um dos corantes mais usados como alvo em estudos de degradação de poluentes orgânicos. Esse é o primeiro estudo que faz uma análise comparativa de modelos cinéticos para degradação oxidativa desse corante por sistemas Fenton. Apesar de vários trabalhos publicados usarem o modelo de $1^{\mathrm{a}}$ ordem, o presente estudo observou que o modelo BMG foi o mais adequado para descolorir esse corante por reação de Fenton clássica $\left(\mathrm{Fe}^{2+} / \mathrm{H}_{2} \mathrm{O}_{2}\right)$. Essa reação se adequou melhor também ao modelo BMG para os outros dois corantes.

Com base nos modelos cinéticos de $1^{\mathrm{a}}$ e $2^{\mathrm{a}}$ ordens, os mediadores fenólicos aumentaram as constantes de velocidade de reação provavelmente ao promover a redução rápida e contínua de $\mathrm{Fe}^{3+}$ a $\mathrm{Fe}^{2+}$ para gerar mais radicais $\mathrm{HO} \cdot{ }^{4}$ Os ácidos 2,3- e 2,5-DHBs foram os mais efetivos mediadores em descolorir esses corantes. $\mathrm{O}$ efeito pro-oxidante desses dois compostos foi mais evidente com o vermelho de fenol, pois esse corante foi o menos suscetível à descoloração. A reação $\mathrm{Fe}^{2+} / \mathrm{H}_{2} \mathrm{O}_{2}$ se adequou bem melhor ao modelo BMG para os três corantes, enquanto a descoloração do azul de metileno também se adequou muito bem ao modelo de $1^{\text {a }}$ ordem. Por outro lado, reações na presença de mediadores se adequaram bem aos três modelos propostos, mas com exceção do modelo BMG para a descoloração do vermelho de fenol ( $\mathrm{r}^{2}$ médio bem inferior a 0,9$)$. A adequação para mais de um modelo cinético também é comum na literatura. A Tabela 3 apresenta alguns modelos estudados para descoloração por sistemas Fenton dos mesmos corantes utilizados no presente estudo, além da descoloração de outros corantes por testar mais de um tipo de modelo cinético. Assim como há trabalhos que fizeram o uso apenas do modelo de $1^{\mathrm{a}}$ ordem, a revisão de Nidheesh et al. destaca que esse é geralmente o escolhido. ${ }^{6}$ Entretanto, os coeficientes de correlação das curvas que descrevem a cinética de algumas reações contendo mediadores para esse modelo são inferiores a 0,9. ${ }^{13,14,18}$ Ao analisar três modelos na descoloração de alaranjado de metila por $\mathrm{Fe}^{2+} / \mathrm{H}_{2} \mathrm{O}_{2}$, Youssef et al. verificaram que nenhum deles apresentou um coeficiente de correlação superior a 0,9. ${ }^{29}$ É importante que seja feita uma análise baseada em diferentes modelos para a determinação dos parâmetros cinéticos, com vistas à obtenção da configuração mais adequada de um reator para degradação de um determinado poluente orgânico por sistemas Fenton. Adicionalmente, vale destacar os dados apresentados pelo grupo de $\mathrm{Xu}$ et al. ao estudar diferentes dosagens de reagentes, catalisadores e corantes..$^{30-32}$ Eles observaram que o modelo cinético mais adequado pode variar de um sistema reacional para o outro, assim como foi verificado nos resultados do presente estudo.

Apesar de o presente trabalho avaliar o efeito de mediadores fenólicos com propriedades redutoras (exceto o ácido 2,4-DHB), os corantes e outros poluentes orgânicos utilizados como alvos podem ser convertidos por radicais $\mathrm{HO}^{\bullet} \mathrm{em}$ intermediários redutores ao longo do tempo reacional, independente da adição de fenóis. A degradação de fenol, 4-clorofenol e 4-nitrofenol por $\mathrm{Fe}^{3+} / \mathrm{H}_{2} \mathrm{O}_{2}$ foi acompanhada pela geração de intermediários di-hidroxilados redutores. ${ }^{36}$ Por outro lado, em um estudo recente envolvendo diferentes sistemas Fenton, a degradação de fenol foi incrementada com a adição de catecol e hidroquinona (1,4-di-hidroxibenzeno), mesmo esses dois tendo sido gerados a partir da hidroxilação da molécula alvo durante as reações. ${ }^{17}$ Por ser um corante muito estudado, produtos de degradação por radicais $\mathrm{HO}^{\bullet}$ do azul de metileno são frequentemente reportados, ${ }^{26,37}$ os quais podem apresentar propriedades redutoras. Embora ele não possua nenhum grupo $\mathrm{OH}$ fenólico, em um estudo anterior do nosso grupo, a presença do azul de metileno aumentou a descoloração do azocorante Pardo Bismarck Y. ${ }^{11}$ Sugeriu-se que o azul de metileno

Tabela 3. Corantes utilizados como alvos em sistemas Fenton publicados na literatura e os modelos cinéticos utilizados para caracterizar as reações

\begin{tabular}{|c|c|c|c|c|}
\hline Corante & Tratamento & Modelos testados & Melhor modelo & Referência \\
\hline \multirow{4}{*}{ Azul de Metileno } & $\mathrm{Fe}^{2+} / \mathrm{H}_{2} \mathrm{O}_{2} ; \mathrm{Fe}^{2+} / \mathrm{H}_{2} \mathrm{O}_{2} / \mathrm{UV}$ & \multirow{3}{*}{$1^{\mathrm{a}}$ ordem } & \multirow{3}{*}{$1^{\mathrm{a}}$ ordem } & 25 \\
\hline & Ferroceno $/ \mathrm{H}_{2} \mathrm{O}_{2}$ & & & 26 \\
\hline & $\mathrm{Fe}^{2+}$-quitosana/ $\mathrm{H}_{2} \mathrm{O}_{2}$ & & & 27 \\
\hline & $\mathrm{Fe}^{2+} / \mathrm{H}_{2} \mathrm{O}_{2}$ & BMG & BMG & 3 \\
\hline \multirow{2}{*}{ Cromotrope $2 \mathrm{R}$} & $\mathrm{Fe}^{2+} / \mathrm{UV} / \mathrm{H}_{2} \mathrm{O}_{2}$ eletrogerado & \multirow{2}{*}{$1^{\mathrm{a}}$ ordem } & \multirow{2}{*}{$1^{\text {a }}$ ordem } & 33 \\
\hline & Eletro-Fenton $\left(\mathrm{H}_{2} \mathrm{O}_{2}\right.$ eletrogerado $)$ & & & 34 \\
\hline Violeta de Metila & $\mathrm{Fe}^{2+} / \mathrm{H}_{2} \mathrm{O}_{2}$ & $1^{\mathrm{a}}$ e $2^{\mathrm{a}}$ ordens, $\mathrm{BMG}$ & BMG & 35 \\
\hline Azul de Argazol & Schorlita/ $\mathrm{H}_{2} \mathrm{O}_{2}$ & $1^{\mathrm{a}}$ e $2^{\mathrm{a}}$ ordens, $\mathrm{BMG}$ & $1^{\mathrm{a}}$ ordem & 30 \\
\hline \multirow{7}{*}{ Alaranjado de Metila } & Schorlita/ $\mathrm{H}_{2} \mathrm{O}_{2}$ & \multirow{6}{*}{$1^{\mathrm{a}}$ e $2^{\mathrm{a}}$ ordens, $\mathrm{BMG}$} & $\mathrm{BMG}$ & 31 \\
\hline & $\mathrm{Fe}_{3} \mathrm{O}_{4} / \mathrm{H}_{2} \mathrm{O}_{2}$ & & $1^{\mathrm{a}}$ ordem & 32 \\
\hline & $\mathrm{Fe}^{2+} / \mathrm{H}_{2} \mathrm{O}_{2}$ & & BMG & \multirow{4}{*}{22} \\
\hline & $\mathrm{Fe}^{2+} / \mathrm{H}_{2} \mathrm{O}_{2} /$ mediadores fenólicos & & BMG, $1^{\text {a }}$ ordem & \\
\hline & $\mathrm{Fe}^{3+} / \mathrm{H}_{2} \mathrm{O}_{2}$ & & BMG & \\
\hline & $\mathrm{Fe}^{3+} / \mathrm{H}_{2} \mathrm{O}_{2} /$ mediadores fenólicos & & $1^{\mathrm{a}}$ e $2^{\mathrm{a}}$ ordens & \\
\hline & $\mathrm{Fe}^{2+} / \mathrm{H}_{2} \mathrm{O}_{2}$ & Zero, $1^{\mathrm{a}}$ e $2^{\mathrm{a}}$ ordens & $2^{\mathrm{a}}$ ordem & 29 \\
\hline
\end{tabular}


possa ter sido convertido em intermediários fenólicos redutores. Para os outros corantes, essa observação também pode ser válida.

Com exceção do ácido 2,4-DHB, o qual foi o menos efetivo mediador por não possuir propriedades redutoras, não foi possível correlacionar a estrutura química dos outros fenóis com suas propriedades pro-oxidantes. Por exemplo, as reações na presença dos compostos redutores contendo grupo carboxila apresentaram maiores valores de $k$ em comparação ao catecol para descolorir o vermelho de fenol. No entanto, o catecol foi um dos mais efetivos mediadores para descolorir os outros dois corantes. Mesmo o ácido gálico possuindo três hidroxilas fenólicas vicinais, grupo funcional envolvido na redução de $\mathrm{Fe}^{3+}, 4$ os ácidos 2,3- e 2,5-DHBs foram os mais efetivos.

É muito importante mencionar que a adição de mediadores fenólicos em sistemas Fenton não é algo simples para o tratamento de efluentes reais, pois o custo desses compostos tornaria esse propósito inviável. Uma alternativa promissora para obtê-los seria utilizar soluções contendo-os e que sejam de fácil disponibilidade. Extratos aquosos de madeira, ${ }^{38}$ assim como um efluente real contendo compostos similares, ${ }^{10}$ incrementaram a degradação de diferentes poluentes orgânicos em sistemas Fenton. Além disso, a toxicidade e a biodegradabilidade desses compostos devem também ser consideradas, caso o efluente tratado seja disposto em corpos receptores e que ainda contenha parte desses fenóis que foram adicionados. Nesse sentido, cabe mencionar que ao reagir com $\mathrm{Fe}^{3+}$, eles são degradados e até mesmo mineralizados ${ }^{23}$ sendo isso algo desejável para aplicação real.

\section{CONCLUSÕES}

Ao comparar os presentes resultados com vários estudos da literatura, o modelo cinético BMG é o que melhor descreve a reação de Fenton clássica para descolorir corantes de diferentes classes. Por outro lado, as reações contendo $\mathrm{Fe}^{3+}$ se adequaram melhor ao modelo de $2^{\mathrm{a}}$ ordem de forma geral, sendo que na presença dos mediadores fenólicos foram observados incrementos nos valores de $k_{2}$ e como consequência a diminuição nos valores de $t_{1 / 2}$. Esses aspectos demonstram que os mediadores aceleraram as reações de descoloração dos três corantes estudados, com exceção do único mediador não redutor, o ácido 2,4-DHB. Esse último apenas apresentou propriedades pro-oxidantes na descoloração do cromotrope $2 \mathrm{R}$. Ainda com base no modelo BMG, a presença dos mediadores fenólicos permitiu incrementos na capacidade oxidativa, assim como na velocidade inicial de descoloração, principalmente para o cromotrope $2 \mathrm{R}$, corante cuja descoloração foi a que melhor se ajustou a esse modelo, independente do estado inicial de oxidação do ferro nos ensaios. Com isso, os três modelos cinéticos permitiram verificar propriedades pro-oxidantes dos mediadores fenólicos. Pode-se corroborar com a literatura que a descoloração oxidativa de corantes por sistemas Fenton pode ser representada por diferentes modelos, sendo influenciada pelo tipo de catalisador, presença ou não de mediador fenólico, assim como da estrutura química do corante alvo.

\section{AGRADECIMENTOS}

Os autores agradecem à FAPEMIG (processos APQ-01585-11 e APQ-01898-17) e ao CNPq pelos auxílios financeiros.

\section{REFERÊNCIAS}

1. Souza, C. R. L.; Peralta-Zamora, P.; Quim. Nova 2005, 28, 226.

2. Oliveira, L. C. A.; Fabris, J. D.; Pereira, M. C.; Quim. Nova 2013, 36, 123.

3. Paulino, T. R. S.; Araújo, R. S.; Salgado, B. C. B.; Eng. Sanit. Ambient. $\mathbf{2 0 1 5}, 20,347$.
4. Aguiar, A.; Ferraz, A.; Contreras, D.; Rodríguez, J.; Quim. Nova 2007, 30,623 .

5. Nogueira, R. F. P.; Trovó, A. G.; Silva, M. R. A. D.; Villa, R. D.; Oliveira, M. C. D.; Quim. Nova 2007, 30, 400.

6. Nidheesh, P. V.; Gandhimathi, R.; Ramesh, S. T.; Environ. Sci. Pollut. Res. 2013, 20, 2099.

7. Santana, C. S.; Aguiar, A.; Water Air Soil Pollut. 2016, 227, 48.

8. Souza, J. L., Aguiar, A.; Rev. Virtual Quim. 2017, 9, 1525.

9. Santana, C. S.; Aguiar, A.; Int. Biodet. Biodegr. 2015, 104, 1.

10. Papoutsakis, S.; Pulgarin, C.; Oller, I.; Sánchez-Moreno, R.; Malato, S.; Chem. Eng. J. 2016, 304, 890.

11. Sousa, J. L.; Aguiar, A.; Environ. Sci. Pollut. Res. 2017, 24, 26734.

12. Christoforidis, K. C.; Vasiliadou, I. A; Louloudi, M.; Deligiannakis, Y.; J. Chem. Technol. Biotechnol. 2018, 93, 1601.

13. Hou, X.; Huang, X.; Ai, Z.; Zhao, J.; Zhang, L.; J. Hazard. Mater. 2016, 310, 170.

14. Li, T.; Zhao, Z.; Wang, Q.; Xie, P.; Ma, J.; Water Res. 2016, 105, 479.

15. Barreto, F.; Santana, C. S.; Aguiar, A.; Desalin. Water Treat. 2016, 57, 431.

16. Friedrich, L. C.; Zanta, C. L. P. S.; Machulek Jr, A.; Quina, F. H.; Quim. Nova 2017, 40, 769.

17. Silva, B. C.; Nogueira, R. F. P.; J. Braz. Chem. Soc. 2016, 27, 1195.

18. Subramanian, G.; Madras, G.; Chem. Commun. 2017, 53, 1136.

19. Levenspiel, O.; Engenharia das Reações Químicas, Edgard Blücher: São Paulo, 2000

20. Behnajady, M. A.; Modirshahla, N.; Ghanbary, F.; J. Hazard. Mater. 2007, 148, 98 .

21. Emami, F.; Tehrani-Bagha, A. R.; Gharanjig, K.; Menger, F.M.; Desalination 2010, 257, 124

22. Santana, C. S.; Velloso, C. C. V.; Aguiar, A.; XXII Congresso Brasileiro de Engenharia Química, São Paulo, Brasil, 2018.

23. Pracht, J.; Boenigk, J.; Isenbeck-Schoter, M.; Keppler, F.; Scholer, H. F.; Chemosphere 2001, 44, 613.

24. Chen, F.; Ma, W. H.; He, J. J.; Zhao, J. C.; J. Phys. Chem. A 2002, 106, 9485 .

25. Melgoza, D.; Hernandez-Ramirez, A.; Peralta-Hernandez, J. M.; Photochem. Photobiol. Sci. 2009, 8, 596.

26. Wang, Q.; Tian, S.; Ning, P.; Ind. Eng. Chem. Res. 2014, 53, 643.

27. Gao, M.; Zhang, D.; Li, W.; Chang, J.; Lin, Q.; Xu, D.; Ma, H.; J Taiwan Inst. Chem. Eng. 2016, 67, 355.

28. Ertugay N.; Acar, F. N.; Arabian J. Chem. 2013, 10, S1158.

29. Youssef, N. A.; Shaban, S. A.; Ibrahim, F. A.; Mahmoud, A. S.; Egypt. J. Pet. 2016, 25, 317.

30. Xu, H. Y.; Liu, W. C.; Qi, S. Y.; Li, Y.; Zhao, Y.; Li, J. W.; J. Serb. Chem. Soc. 2014, 79, 361.

31. Xu, H. Y.; Shi, T. N.; Wu, L. C.; Qi, S. Y.; Water Air Soil Pollut. 2013, 224,1740

32. Xu, H. Y.; Wang, Y.; Shi, T. N.; Zhao, H.; Tan, Q.; Zhao, B. C.; He, X. L.; Qi, S. Y.; Front. Mater. Sci. 2018, 12, 34.

33. Almeida, L. C.; Garcia-Segura, S.; Arias, C.; Bocchi, N.; Brillas, E.; Chemosphere 2012, 89, 751.

34. Almeida, L. C.; Silva, B. F.; Zanoni, M. V. B.; J. Eletroanal. Chem. 2014, 734, 43 .

35. Ou, X.; Wang, C.; Zhang, F.; Sun, H.; Wuyunna; Desalin. Water Treat. 2013, 51, 2536.

36. Du, Y.; Zhou, M.; Lei, L.; J. Hazard Mater. 2006, B136, 859.

37. Oliveira, L. C. A.; Gonçalves, M.; Guerreiro, M. C.; Ramalho, T. C.; Fabris, J. D.; Pereira, M. C.; Sapag, K.; Appl. Catal. A Gen. 2007, 316, 117.

38. Aguiar, A.; Ferraz, A.; Int. Biodet. Biodegr. 2012, 74, 61. 